\title{
A SPICE IGBT Model with easy parameters extraction
}

\author{
Hadini Yassine ${ }^{1}$, Galadi Abdelghafour ${ }^{2}$, Echchelh Adil $^{3}$ \\ \{yassine.hadini@uit.ac.ma1,agaladi@yahoo.fr ${ }^{2}$, adilechel@gmail.com ${ }^{3}$ \} \\ ${ }^{1,3}$ Laboratory of Electrical Engineering and Energy System, Department of physics Faculty of Sciences, \\ Ibn Tofail University, Kenitra, Morocco \\ ${ }^{2}$ Team: Electronic, Electrical engineering, Instrumentation and Imaging, National School of \\ Applied Sciences, Cadi Ayyad University, Safi, Morocco
}

\begin{abstract}
An accurate IGBT model is presented in this paper. It is based on the IGBT physical structure. The model uses an equivalent circuit which combines a MOSFET, controlled by a voltage-controlled voltage source, in series with a diode. the required parameters can be extracted with a simple procedure from the datasheet dc characteristics given by the manufacturers. The results of the comparison between the simulation and the datasheet information shows an average error less than $9 \%$ for the output characteristics and less than $7 \%$ for the transfer characteristic. Moreover, the model is simple and can be implemented in any SPICE-based circuit simulator. A description of the model and the parameter extraction procedure will be provided along.
\end{abstract}

Keywords:IGBT, SPICE, MODEL, MOSFET, DIODE.

\section{Introduction}

In power electronic circuit, the power transistors are used as a switch to control the power electric energy. To sustain high voltage in the off-state, the power transistor structure contains a thick and lowly doped region called the drift region [1]. In case of the power metal-oxidesemiconductor field-effect transistor (MOSFET), the drift region turns into high resistance restricting the power electronic applications of this type of devices. In bipolar power transistor, the resistance of the drift region is reduced considerably thanks to the modulation of the conductivity of this drift region [1]. The insulated gate bipolar transistor (IGBT) transistor structure (Figure 1), is a hybrid MOSFET-bipolar configuration which is equivalent to an bipolar current controlled by an MOSFET. Thanks to the high input impedance of the MOSFET, the IGBT is controlled by a low gate drive power. As IGBT voltage and current ratings increase, its application range is extending to high power applications; that is why, in the very near future, the IGBT will be a serious competitor to the GTO [2], [3].

On the other hand, the peculiar IGBT structure makes the SPICE modeling of the IGBT a very complex task. In literature, the IGBT model has been represented by a couple of a simplified equivalent circuit. The first presents the IGBT as a N-channel MOSFET driving a PNP bipolar transistor in a Darlington configuration [4]-[8], whereas the second consists of an $\mathrm{N}$ channel MOSFET in series with a diode [9]. The proposed models cannot describe the IGBT behaviour accurately, because the wide base in the IGBT differs from the existing discrete power BJT model. Furthermore, these models cannot extract the BJT current gain BF parameter from 
the datasheet information like all the other parameters used in the model. While Mihaliç et al [7] chose the maximum possible value for $\mathrm{BF}$ parameter as $\mathrm{BF}=100$, Kim et al [5] assumed the current gain $\mathrm{BF}$ equal to 1 .

In this paper, an IGBT equivalent circuit, based on an N-channel MOSFET in series with diode and effective voltage controlled voltage source (VCVS) is proposed. All needed parameters of the model are extracted easily by using a simple method from the measurements. The MOSFET model parameters are extracted from the transfer characteristic, while the diode parameters are extracted from the output characteristics. Moreover, our model takes into consideration the IGBT transconductance flattening out [10] which occurs in high gate voltage, by VCVS in the MOSFET gate. The validity of this model has been confirmed by the comparison between the simulation and the measurements given in the manufacturer's datsheet for the IGBT CM600HA-24H. The comparison shows that the proposed model gives $9 \%$ as an average error for the output characteristics and $7 \%$ for the transfer characteristic, the DC model description will be given in section 2, the parameters extraction procedure will be explained in section 3 , the results of the comparison between the measurements and simulation will be presented in section 4 and the conclusion of this paper is given at the end.

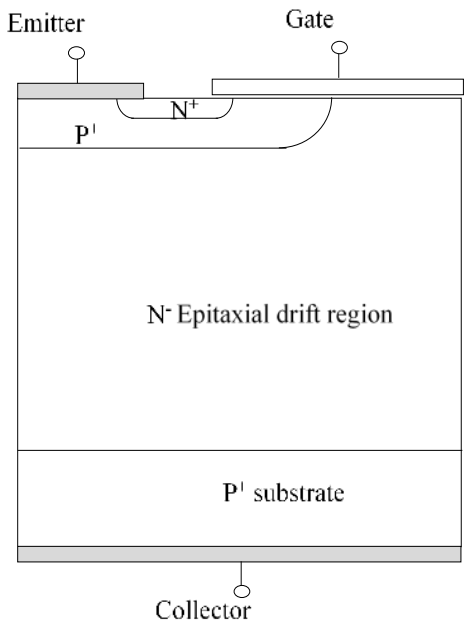

Fig 1. Cross-sectional view of NPT IGBT structure

\section{Model description}

As in the MOSFET, the IGBT collector current is controlled by the applied gate voltage VGE. When this applied voltage exceeds the threshold voltage (Vth), a channel is created underneath the gate allows the current to flow. The output characteristics show the liaison between collector voltage, collector current and the gate voltage. They are different from those of the MOSFET as they have a cut-off region where the output current is zero [11].Between the output voltage and the saturation voltage, the IGBT operates in the linear region, where the collector voltage is determined by the collector current and the gate voltage. Above the saturation voltage up to breakdown voltage, IGBT operates in the saturation mode. The transfer characteristic and the gate voltage determine the collector current at any collector voltage [12]. 


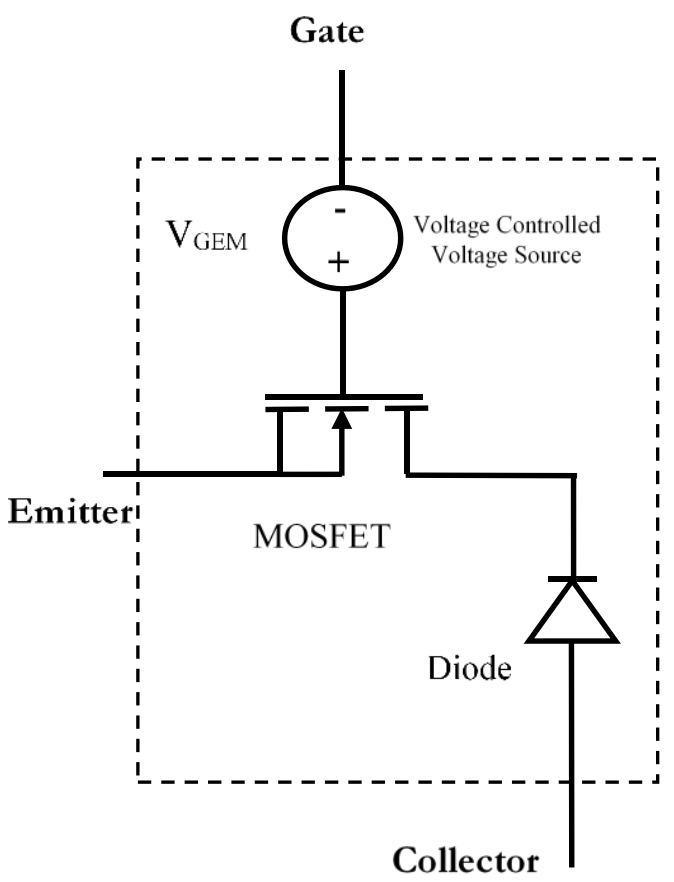

Fig 2. Schematic of the proposed model for the IGBT

Based on the description presented before, and on the physical structure, the proposed model uses a channel intrinsic MOSFET controlled by a Voltage Controlled Voltage Source (VCVS), in series with a diode as shown in Figure 2 to model the IGBT behaviour in its different states (cut-off, linear and saturation).

The intrinsic MOSFET is used to represent the effect of the channel created under the gate as described above. Therefore the MOSFET model describes the IGBT behaviour in saturation region and also in a part of the linear region [13]. The SPICE MOSFET model level 1 has chosen for this task, because of its simplicity and the reduced number of its parameters. The channel transconductance $(\mathrm{Kp})$ and the threshold voltage $(\mathrm{Vth})$ are the essentials parameters to model accurately the IGBT behaviour in these regions. Then the current in these regions has the following equations:

$$
I\left\{\begin{array}{ccc}
= & 0 \\
& \text { if } \quad V_{G E}<V_{t h} \quad \text { or } \quad V_{C E}<V_{D} \\
= & K_{P}\left[\left(V_{G E}-V_{t h}\right)\left(V_{C E}-V_{D}\right)-\frac{\left(V_{C E}-V_{D}\right)}{2}\right] \\
& \text { if } \quad V_{G E} \geq V_{t h} \quad \text { and } \quad V_{C E}<V_{G E}+V_{D}-V_{t h} \\
= & \frac{K_{p}}{2}\left(V_{G E}-V_{t h}\right)^{2} \\
& \text { if } \quad V_{C E} \geq V_{G E}+V_{D}-V_{t h}
\end{array}\right.
$$

The diode is used to model the IGBT behaviour in the cut off region and the beginning of the linear region. And the current is modelled by the Diode SPICE model equation: 
$\mathrm{I}=\mathrm{I}_{\mathrm{S}}\left[\exp \left(\frac{V_{D}}{N V_{T}}\right)-1\right]$

The two basic required parameters for the diode model are the saturation current Is and the emission coefficient $\mathrm{N}$.

The sub-circuit composed, just, by a MOSFET in series with a diode, could not describe accurately the IGBT behaviour in the saturation region, especially in the high VGE. Because the MOSFET SPICE model level 1 could not model the drop of the IGBT transconductance due to the channel saturation in the high VGE. So, to eliminate the difference between the simulation and the measurement in the saturation region, the VGE applied in the model should be less than the VGE used in the measurements for the same IC value. Therefore, to overcome this disagreement, a voltage-controlled voltage source (VCVS) is added. The main role of the VCVS is the control of the gate voltage, in the purpose to compensate the difference existed between the real VGE value and this applied in the model. the VCVS depends linearly on the VGE as described in the following equations:

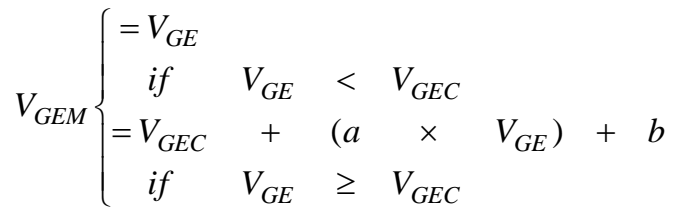

When

$$
f\left(V_{G E}\right)=a \times V_{G E}+b(4)
$$

VGEC is a critical gate-emitter voltage at the onset of the transconductance drop phenomenon happened in the high VGE. a and $\mathrm{b}$ are the VCVS parameters.

Our proposed model uses the sub-circuit approach based on the physical structure to describe the IGBT behaviour. The new proposed IGBT model is easy to implement in the PSPICE either as sub-circuit or as a compact model using a netlist. Also, all required parameters of the model are simply extracted from the experimental results given in the manufacturer datasheet.

\section{Parameter extraction}

\subsection{MOSFET parameters}

The basic parameters of this model are the transconductance Kp and the threshold voltage Vth. The two parameters are extracted from the transfer characteristic in high VCE.

The MOSFET channel controls the IGBT behaviour in saturation region. the saturation region of the MOSFET and the IGBT can be assumed to be the same, therefore they have the same transfer characteristic in high VCE. For this etude the VCE chosen is $10 \mathrm{~V}$. the level 1 SPICE model is used to model the MOSFET, then the MOSFET drain current in the saturation region is given by the following equation:

$$
I=\frac{K_{p}}{2}\left(V_{G E}-V_{T H}\right)^{2}
$$

From the linear portion of the transfer characteristic, we trace a straight line whose equation has the following form: 


$$
\sqrt{I}=\sqrt{\frac{K_{P}}{2}} V_{G E}-\sqrt{\frac{K_{P}}{2}} V_{T H}
$$

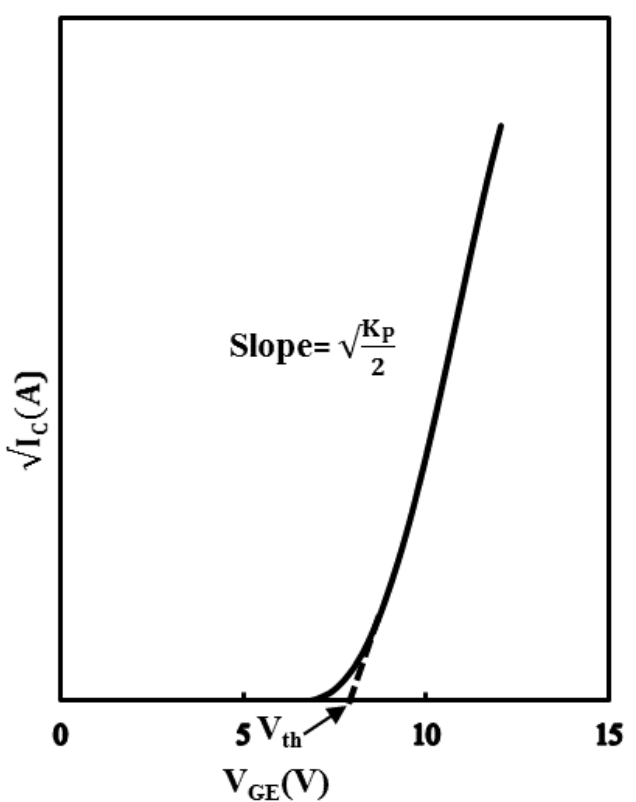

Fig 3. Square root of collector current vs gate voltage defines $V_{\text {th }}$ and $K_{P}$.

$\mathrm{KP}$ can be determined from the slope factor of the $\sqrt{\mathrm{IC}}-\mathrm{VGE}$ curve, while Vth is the intersection between the extension of the linear portion and the VGE-axis as shown in Figure 3.

\section{2 . Diode parameters}

The saturation current Is and the ideality factor $\mathrm{N}$ are considered as the essential parameters required to model the diode. They are extracted from the output characteristics in low collector voltage and high gate voltage. The diode controls the IGBT behaviour when VCE is in vicinity of the junction PN cut-in voltage.

$\exp \left(\frac{V_{C E}}{N V_{t}}\right) \gg 1$ then the equation (2) may be simplified

$$
\ln I=\ln I_{S}+\frac{V_{C E}}{N V_{t}}
$$

Is and $\mathrm{N}$ can be extracted from the equation (7).

\subsection{The voltage controlled voltage source parameters}

\subsubsection{Critical voltage $V_{\text {GEC }}$}

In order to determine the critical VGE (VGEC). We trace the IC-VGE curve. from it we can define the VGEC as shown in the figure 4. 


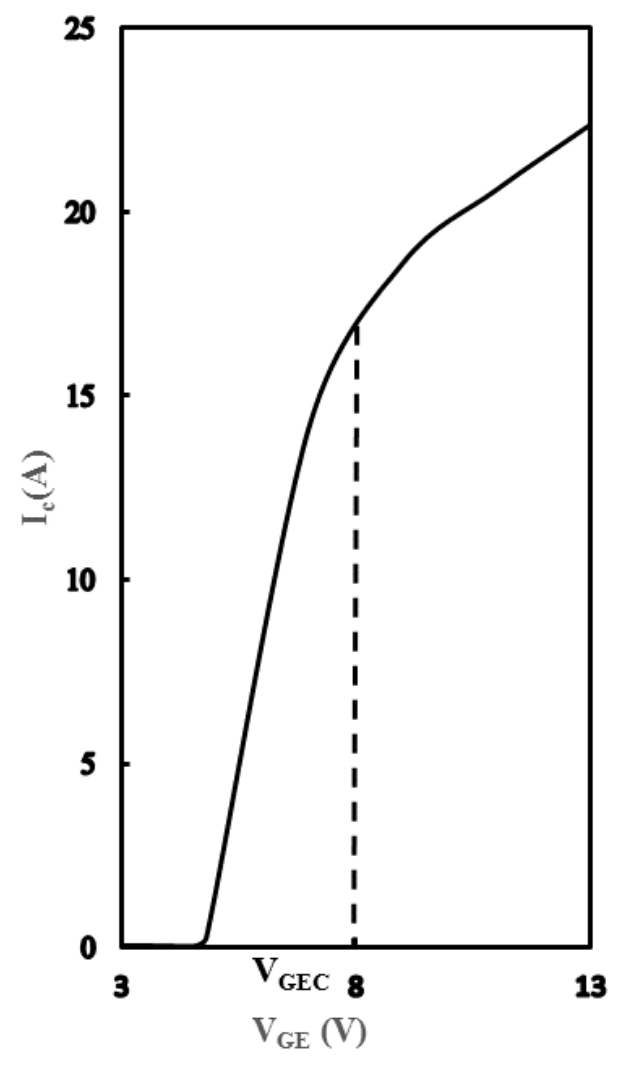

Fig 4. Collector current vs gate voltage defines $\mathrm{V}_{\mathrm{GEC}}$

\subsection{2 a and b VCVS parameters}

When VGE > VGEC, the VGEM can be given by:

$$
V_{G E M}=\frac{I_{C}}{K_{P}\left(V_{C E}-V_{D}\right)}+\frac{\left(V_{C E}-V_{D}\right)}{2}+V_{t h}
$$

Where VD is the voltage drop in the diode calculated by the following equation

$$
V_{D}=N V_{T} \times \ln \left(\frac{I_{C}}{I_{S}}\right)
$$

Then from the equations (3), (4) and (8) a and b can be determined.

All the proposed methods in this section are verified by comparison with the experimental results given in datasheet. The comparison between simulated and experimental results will be shown and discussed in the following section. 


\section{Results and discussion}

After having determined the parameters of the model, using the methods described above. The sub-circuit is simulated using PSPICE in order to compare the simulation results of the dc analysis with the dc characteristics given in the datasheet.

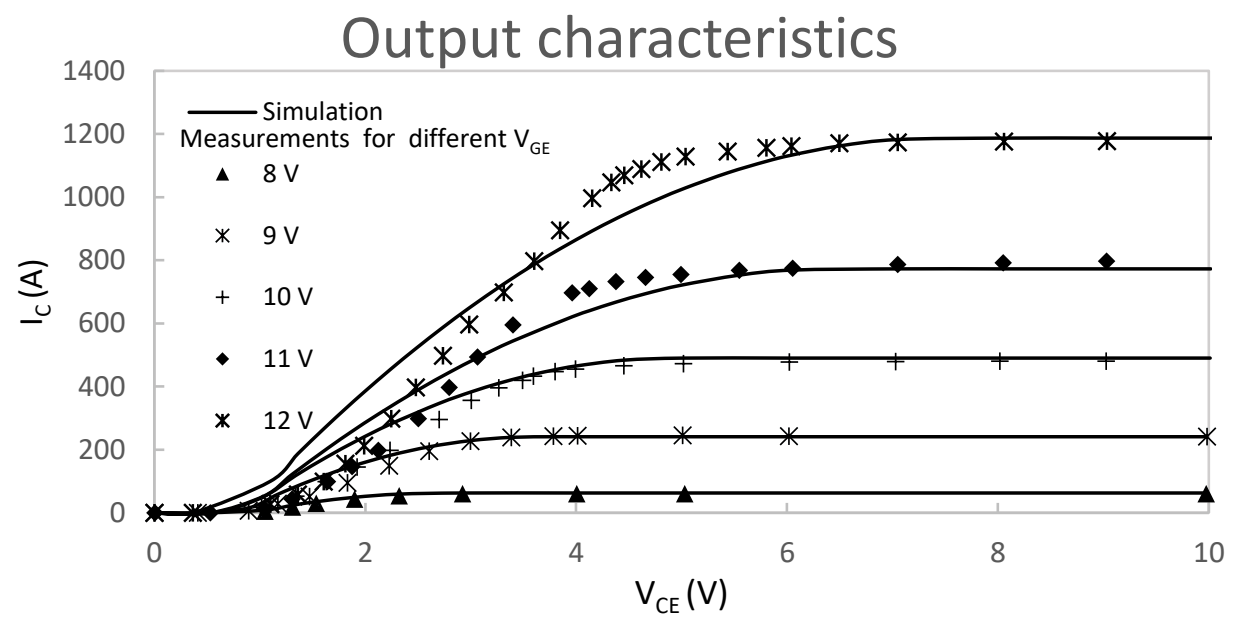

Fig 5. Measured output characteristics in comparison to the proposed model applied to the IGBT CM600HA-

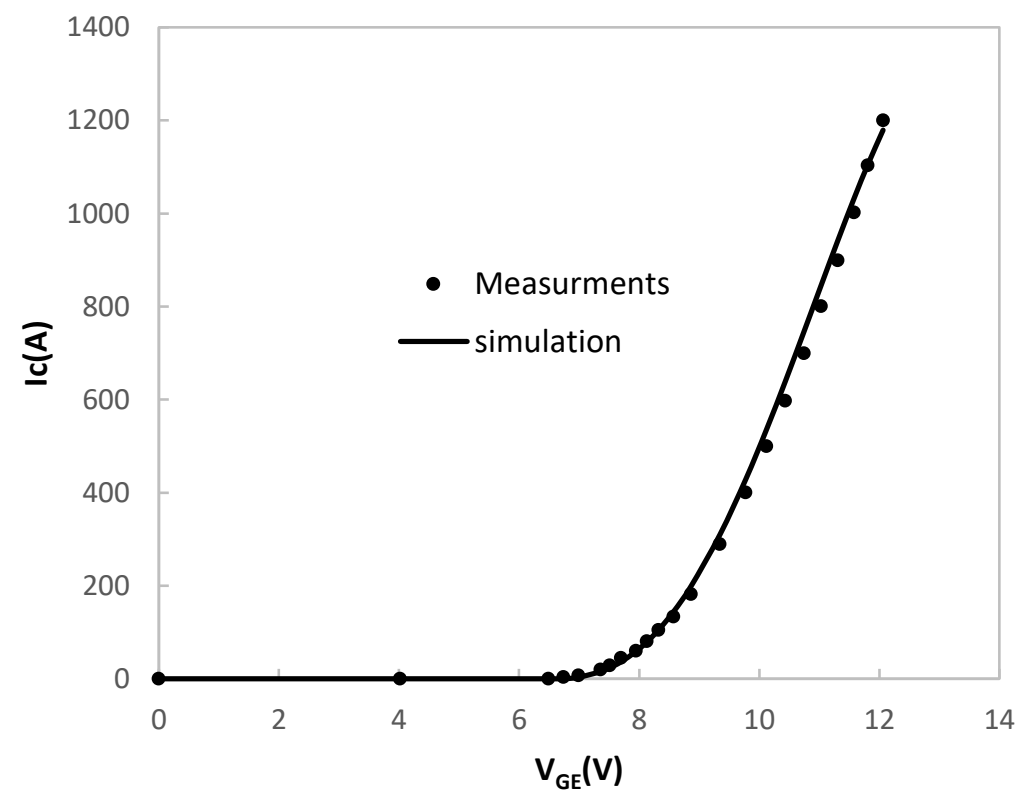

Fig. 6. Measured transfer characteristic in comparison to the proposed model applied to the IGBT CM600HA-24H for $\mathrm{V}_{\mathrm{CE}}=10 \mathrm{~V}$. 
While the Figure 5 shows the measured output characteristics in comparison with the simulation result of the proposed model applied to the IGBT CM600HA-24H, the Figure 6 shows the measured transfert characteristic in comparison to the proposed model applied to the IGBT CM600HA-24H for VCE $=10 \mathrm{~V}$.

The proposed model presents a good agreement with the experimental results. The average error is less than $9 \%$ for in output characteristics and less than $7 \%$ in transfer characteristic.

It can be observed that in the linear region the measurement is a little higher than the simulation, this difference is due to two main causes, the first is that the model of the diode is unable to precisely describe the fort level injection presents in the IGBT N-base region [14], the second is that the transition from the linear region to the saturation region in the IGBT differs from which in the MOSFET SPICE level 1 model used in this work [11]. For this reason, the model proposed in this work can be used only for the NPT IGBT, where the level of injection in the base region is less important than the other IGBT types.

\section{Conclusion}

In this paper, the IGBT SPICE macromodel has been described. The static I-V characteristics offered by the macromodel are shown and the model efficiency is demonstrated by the comparison between the simulated and experimental results of two IGBT devices. The parameters extraction method is represented and all parameters such as Vth, KP, Is and $\mathrm{N}$ are successfully obtained from the datasheet characteristics. Our model is simple and easy to be implemented in any SPICE-based circuit simulator.

The future research direction could have as purpose, the improvement of this model to describe accurately all kind of IGBT.

\section{References}

[1] B. J. Baliga, The IGBT device: physics, design and applications of the insulated gate bipolar transistor. Kidlington, Oxford ; Waltham, MA: William Andrew/Elsevier, 2015.

[2] K. Sheng, B. W. Williams, and S. J. Finney, "A review of IGBT models," Power Electron. IEEE Trans. On, vol. 15, no. 6, pp. 1250-1266, 2000.

[3] S. Yuan, S. Yuan, Y. Hong, and Y. Liu, "SPICE Model for IGBT Temperature Effects Compared with Measurements," in 2010 International Conference on Electrical and Control Engineering, 2010, pp. 3037-3040.

[4] K. Asparuhova and T. Grigorova, "IGBT high accuracy behavioral macromodel," in Microelectronics, 2008. MIEL 2008. 26th International Conference on, 2008, pp. 185-188.

[5] H. S. Kim, Y. H. Cho, S. D. Kim, and Y. I. Choi, "Parameter extraction for the static and dynamic model of IGBT," in , 24th Annual IEEE Power Electronics Specialists Conference, 1993. PESC '93 Record, 1993, pp. 71-74.

[6] L. Michel, A. Chériti, and P. Sicard, "Development of an efficient IGBT simulation model," in Electrical and Computer Engineering, 2009. CCECE'09. Canadian Conference on, 2009, pp. 252-256.

[7] F. Mihalič, K. Jezernik, K. Krischan, and M. Rentmeister, "IGBT spice model,” Ind. Electron. IEEE Trans. On, vol. 42, no. 1, pp. 98-105, 1995.

[8] H.-S. Oh and M. El Nokali, "A new IGBT behavioral model," Solid-State Electron., vol. 45, no. 12, pp. 2069-2075, Dec. 2001.

[9] "IGBT tutorial: Part 1 - Selection | EE Times," EETimes. [Online]. Available: http://www.eetimes.com/document.asp?doc_id=1273173. [Accessed: 23-Jul-2016]. 
[10] V. K. Khanna, Insulated Gate Bipolar Transistor IGBT Theory and Design. John Wiley \& Sons, 2004.

[11] K. Asparuhova and T. Grigorova, "IGBT behavioral PSpice model," in Microelectronics, 2006 25th International Conference on, 2006, pp. 203-206.

[12] I. Baraia, J. Galarza, J. A. Barrena, and J. M. Canales, "An IGBT behavioural model based on curve fitting methods," in Power Electronics Specialists Conference, 2008. PESC 2008. IEEE, 2008, pp. 1971-1977.

[13] P. Igić and N. Janković, "Review of advanced IGBT compact models dedicated to circuit simulation," Facta Univ.-Ser. Electron. Energ., vol. 27, no. 1, pp. 13-23, 2014.

[14] B. J. Baliga, "Analytical Modeling of IGBTs: Challenges and Solutions," IEEE Trans. Electron Devices, vol. 60, no. 2, pp. 535-543, Feb. 2013. 